\title{
SDL+QualNet: A Novel Simulation Environment for Wireless Heterogeneous Networks
}

\author{
Pero Latkoski, Valentin Rakovic, Ognen Ognenoski, Vladimir Atanasovski, Liljana Gavrilovska \\ Faculty of Electrical Engineering and Information Technologies \\ Ss. Cyril and Methodius University \\ Skopje, Macedonia \\ \{pero,valentin,ognen,vladimir,liljana\}@feit.ukim.edu.mk
}

\begin{abstract}
The development of new network communication protocols has to include a process of concept evaluation in order to prove its soundness. Simulation-based techniques provide assessment of communication quality in complex and realistic working conditions. This paper introduces a novel and unique simulation technique, by merging QualNet simulator and SDL protocol developer. Many complex modifications of the QualNet nodes can be efficiently implemented, which is very difficult by a straightforward re-coding of QualNet components. The proposed methodology was utilized in the development of a wireless heterogeneous IEEE 802.21-based network. The paper provides a detailed insight of the model's three basic parts: QualNet environment, SDL resource manager and the novel SDL2QualNet interface. We present useful information for 802.21 simulation implementation, data acquisition from QualNet and calculation of dynamic network parameters, information and buffer management, methods for improvement of simulation efficiency, and many other modeling hints which enable a comprehensive simulation platform.
\end{abstract}

\section{Categories and Subject Descriptors}

C.2.1 [Computer-Communication Networks]: Network Architecture and Design - Network communications, Wireless communication.

\section{General Terms}

Performance, Design, Reliability, Verification.

\section{Keywords}

Heterogeneous networks; Modeling; SDL; QualNet;

\section{INTRODUCTION}

Each newly designed network concept needs to be put under a reliability and validity evaluation process. Estimation of network performance is another issue which must be addressed.

Permission to make digital or hard copies of all or part of this work for personal or classroom use is granted without fee provided that copies are not made or distributed for profit or commercial advantage and that copies bear this notice and the full citation on the first page. To copy otherwise, or republish, to post on servers or to redistribute to lists, requires prior specific permission and/or a fee.

SIMUTools 2010 March 15-19, Torremolinos, Malaga, Spain.

Copyright 2010 ICST, ISBN 78-963-9799-87-5.
Analytical modeling, numerical calculations and simulationbased performance evaluation are the basic approaches to evaluate the quality of the developed communication networks and protocols. The analytical and numerical analysis usually requires a certain amount of problem simplification and a controlled introduction of approximations. On the other hand, simulations-based method provides an evaluation of communications system's performance in far more realistic scenarios. The only condition in this case is to prepare a suitable simulation environment and to build a comprehensive simulation model. Today, the research society employs different modeling and simulation tools and methods. Some of them are suitable for building comprehensive communication network scenarios and some for a rapid communication protocol development. The former type of modeling tools usually provides a wide range of precompiled radio access technology packages, along with a variety of channel and mobility models, routing and session management protocols, as well as application emulators. The later type of simulation and modeling tools is suitable for protocol procedure development and provides means for building an unambiguous specification of the processes inside the communication protocol layers.

In order to provide a comprehensive simulation model of a communication network, it is preferable to combine two or more developing tools and methods [3]. This paper presents a novel and powerful simulation environment used for wireless networks modeling. It is applied on the RIWCoS architecture $[15,8]$ and its mechanisms for handling applications and network resources. The analyzed communication problem of wireless networks integration gains momentum among network developers and network operators. The idea behind is to create a single heterogeneous communicating environment which will provide a seamless horizontal and vertical handover to its users $[11,13,12]$. Recently, efforts are made to develop terminal equipment which will be able to exploit the benefits of the network heterogeneity $[10,6]$. The introduction of intelligent resource management ability into the network is a challenging task. One way of fulfilling this goal is to introduce necessary amendments to the existing wireless technologies, along with a standardized agreement which will provide a common interoperability umbrella. The IEEE 802.21 standard is the most promising candidate for this purpose [7].

The paper is organized as it follows. Section 2 explains the communication model and the RIWCoS concept. Section 3 contains the reasons for choosing QualNet as a simulator in which the network simulation scenario is created, and the 
arguments for utilizing the Specification and Description Language (SDL). Section 4 provides details of the network model implementation, whose evaluation is presented in the following Section 5. Finally, the paper draws its conclusions in Section 6.

\section{MODEL DESCRIPTION}

\subsection{Wireless Heterogeneous Networks}

Development and implementation of functional wireless heterogeneous networks (WHN) is an aspect of paramount importance that inspires academia research and industry for delivering next generation services to the end users. The coexistence of the variety wireless access technologies in unified $4 \mathrm{G}$ platform provides wide range of possibilities for delivering services anywhere and anytime to mobile users. In order to preserve the quality of service for such users, the paradigm "seamless mobility" must be provided. It denotes the situation when ongoing sessions are maintained with minimal performance degradation while the user transits in the heterogeneity of the communication system, and establishing sessions via different access technologies. This feature can be provided only by achieving interoperability between the networks and reconfiguration of the communication protocol stack inside the communication entities. The interoperability feature provides unification of the different wireless access technologies and provides establishment of sessions on multiinterface terminals depending on the application requirements and network conditions. The reconfigurability feature makes the decision on the type of resource and network access that should be used in the interoperable system, in order to deliver the service with an appropriate quality. During the reconfiguration, the system must allocate resources in fast and efficient manner, in order for the reconfigured session to preserve its quality. The wireless resources significantly change in time, so the schemes for resource allocation in this sense must be carefully designed in order to meet application demands before, after and during the reconfiguration.

\subsection{RIWCoS Concept}

The Reconfigurable Interoperability of Wireless Communication Systems (RIWCoS) concept denotes the design and development of a system that integrates variety of wireless technologies (e.g. WLAN, WiMAX) along with the implementation of interoperability (IM) and resource management (RM) modules. The RIWCoS system proposes distributed architecture with modules allocated in each wireless point of attachment (PoA) in the heterogeneous environment and in each RIWCoS terminal. The functionality of the modules combined with the communication protocols implemented between them enables reconfigurable interoperability for the sessions started on the RIWCoS terminals. There are also modules proposed for the core network that are on a higher hierarchical level which monitor the entire network topology.

The interoperability of the component wireless technologies within the RIWCoS is achieved according to IEEE 802.21 standard. This standard proposes new entity and new protocol design for Media Independent Handover (MIH) that provides seamless mobility for the MIH users. The Media Independent Handover Function (MIHF) is an entity for abstracting the

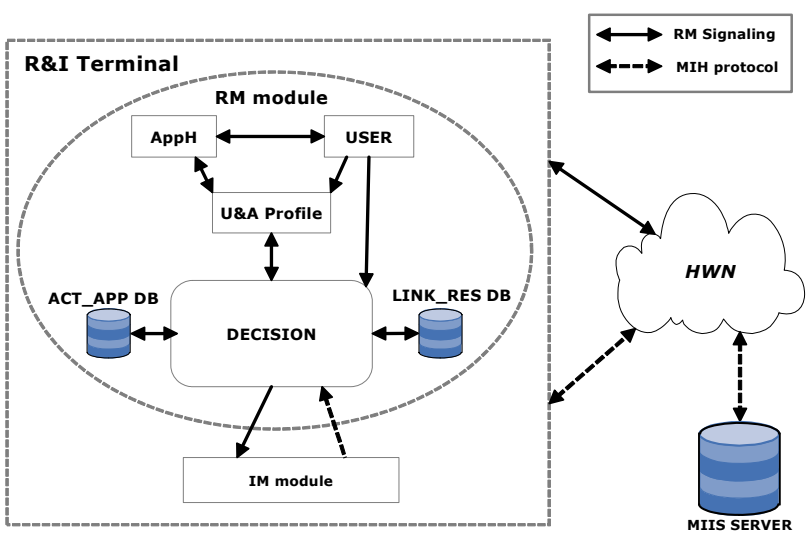

Figure 1. The RIWCoS terminal RM module

media dependency to MIH users. The standard provides the system interoperability by specifying a set of events, commands and information that define the MIH protocol. The reconfiguration in the system is done with implementation of distributed Resource Management modules that are developed as MIH users. These modules obtain information from the IM modules about the network conditions (resource availability) and issue decision commands about the selected resource allocation depending on the application requirements.

The RM in RIWCoS aims to achieve fast and effective resource allocation using the underlying interoperability provided with the MIHF. The resource management system consists of distributed modules that can be grouped in three categories according to the position in the system:

- $\quad \mathrm{RM}$ module in the terminal;

- $\quad$ RM module at each PoA;

- RM module in the core network.

The structure of the terminal RM module is depicted in Fig. 1. It shows the building blocks and the interactions between the building blocks (one way and two way communication). The structure of the rest of the modules is based on the logical building entities as in the terminal RM. Their functionality differs depending on the type of the PoA and the design of the core network. The functionality of the RM system in the interoperable heterogeneous environment is supported with the 802.21 defined Media Independent Information Server - MIIS. Short elaboration of the RM's building entities follows in order to present its functionalities. This is essential for the development of the module in the simulation environment.

AppH: block for handling the user applications. It communicates with the User and User \& Application (U\&A) Profile and delivers information for the started applications on the terminal. It collects the information of all initiated applications from the appropriate user and can provide demand reconfiguration if needed. The application requests in general are described with the needed bitrate and the delay that the network resources should provide. In addition, the application handler determines the minimal set of bitrate and maximum delay for each application in order to reconfigure the application demands (hence, decrease its QoS) when there is a high traffic in the communication system. This makes the system more flexible for implementation in emergency scenarios, where there 
is a failover of some technology. Reconfiguration of application demands can be useful for providers when load balancing is needed.

User: block for defining the user type. User classification in the future wireless system will be imminent due to the enlarged possibilities for personalization provided by the WHN. In the RIWCoS system the users are classified in three categories according to their service level agreement (gold, silver and bronze). The type of the user determines the reconfiguration decision in the RM module and drives the work of the Decision block.

U\&A Profile: the block that combines the user type and its application demands. This block sends message for the type of user and the characteristics of each started application. The message is passed to the Decision block which decides about the resource allocation from the available network resources.

Decision: core block of the RM module. This block collects the information about the user and the underlying technologies. It stores the MIH data in the database LINK_RES DB. The information stored in this database is dynamically updated with the events received from the IM module. For each user application, depending of the message from the U\&A Profile the Decision will allocate link resource from LINK_RES DB. When this is done, the database will be updated, and a query will be sent to ACT_APP DB database in which the active application will be stored. When an application finishes the session, the entry will be deleted by the Decision Block. The Decision uses two types of data: static and dynamic. The MIIS server provides info about the network topology and the network resources. This information should be of more static nature, like cost of the network, type of the network (e.g. IEEE 802.11b), implemented network policies, etc. In contrast to this static information, the terminal should have dynamic information from the appropriate PoA (e.g. link condition): BITRATE supported on the link and the AVERAGE_PCK_DELAY of the packets. This information is obtained via $\overline{\mathrm{M} I H}$ commands.

\subsection{IEEE 802.21 Role}

The role of the IEEE 802.21 standard $[16,1]$ is essential for efficient functionality of the RM module, because it provides the interoperability of the communication system. The RM module uses the semantics of the standard along with the messages and commands that are obtained from the network via the IM module. The decision commands that are issued towards the IM module should be standard defined as the target destination can be local or remote network entity. The set of messages that are used from the standard for obtaining the needed information are: MIH_Capability_Discover.request, MIH_Capabilit_Discover.confirm, MIH_Link_Get_Information.request, MIH_Link_Get_Inform.confirm, MIH_Link_Get_Params.request and MIH_Link_Get_Params.confirm. These messages are obtained from the $\overline{M I H F}^{-}$via the standard defined MIH_SAP. The decisions issued from the RM module are sent through the same MIH_SAP and are enforced by the IM modules in the RIWCoS system.

\section{SDL\&QualNet Simulation Platform}

The proposed integration of SDL and QualNet represents a powerful simulation environment which can be used for rapid implementation of new resource management procedures and protocols. The following section describes the actual realization of the SDL+QualNet simulation platform used for the development and analysis of wireless heterogeneous networks model.

\subsection{SDL Protocol Descriptor}

The development of communication protocols and protocol entities is a complex engineering task. It begins with capturing of communication requirements and entities' behavior, continues with the performance prediction, design and implementation and ends with the conformance testing. Traditionally the development of communication protocols is performed in an informal way by using a straightforward programming and code production, based on an informal textual documentation and graphical description. This method lacks of a scientific rigorousness and leads to an ambiguous implementation of the requirements. The conformance testing in this case is based on heuristic queries with a limited coverage.

The introduction of the formal description techniques (FDTs) has brought correctness and reliability into the protocol development process. The need for FDTs has been recognized a long time ago $[14,12]$. Today there are many formal methods used in the protocol development process: Specification and Description Language (SDL) [17], Simple ProMeLa Interpreter (Spin) [24], Estelle [18], Language of Temporal Ordering Specifications (LOTOS) [19], Petri Nets [9], Uppaal [4], Message Sequence Chart [20] and Unified Modeling Language [21]. Among them, SDL has achieved widespread success because of its friendly graphical notation, its standardization by the International Telecommunication Union (ITU-T) as the major standards specification tool, and its support for other popular notations such as ASN.1 [22], MSC and TTCN [23]. The effectiveness of SDL and its ability to develop unambiguous protocols [5] have won it a widespread popularity and have led the standardization institutes, such as, ETSI (European Telecommunications Standards Institute) [25], 3GPP (Third Generation Partnership Project) [26] and IEEE (The Institute of Electrical and Electronics Engineers) [27] to include SDL diagrams in their protocols specification. Because of the previously stated advantages, SDL was selected as a protocol description method for the purpose of our analysis.

The SDL part of the RIWCoS model provides multiple functionalities to the simulation process of a wireless heterogeneous network. Its main role is the specification of protocol layers and procedures regarding the RIWCoS resource manager which need to be implemented in each network node. Additionally, all IEEE 802.21-defined entities are included in the SDL part of the model. A common radio resource manager (CRRM) of the heterogeneous network embedded in the SDL part completes its general architecture. All these functionalities are developed as a separate SDL blocks inside the global SDL system level of specification (Figure 2). As depicted in Figure 2, there is only one CRRM block, and one MIIS block. On the other hand, RIWCoS resource manager is created as a SDL Block Type (RIWCoS_Type), which is later instantiated several times (in the presented case, 11 times), depending on the number of nodes defined in the QualNet's simulation scenario. Each QualNet node corresponds to one instance of the RIWCoS RRM. The instances are formally identical, but independent of 


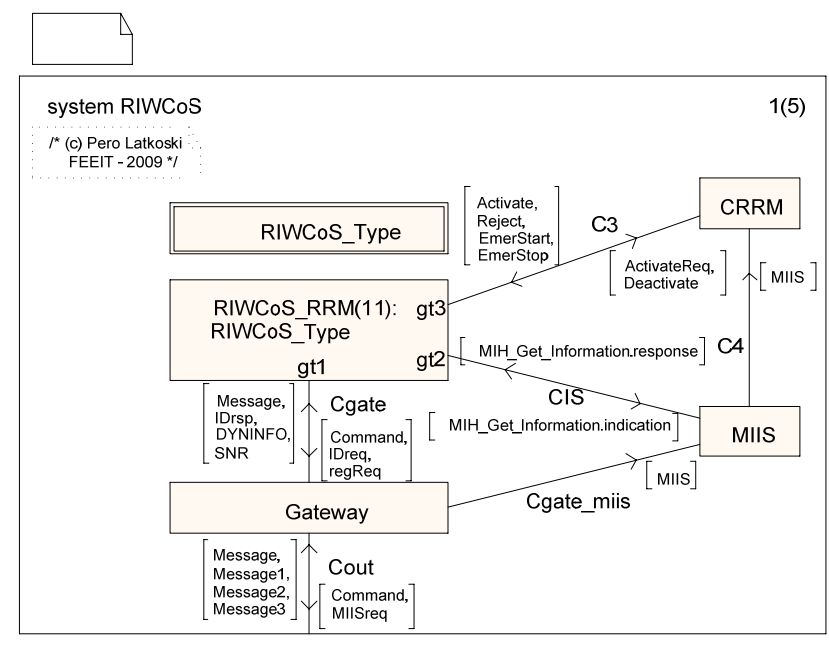

Figure 2. SDL part of the RIWCoS model (system level)

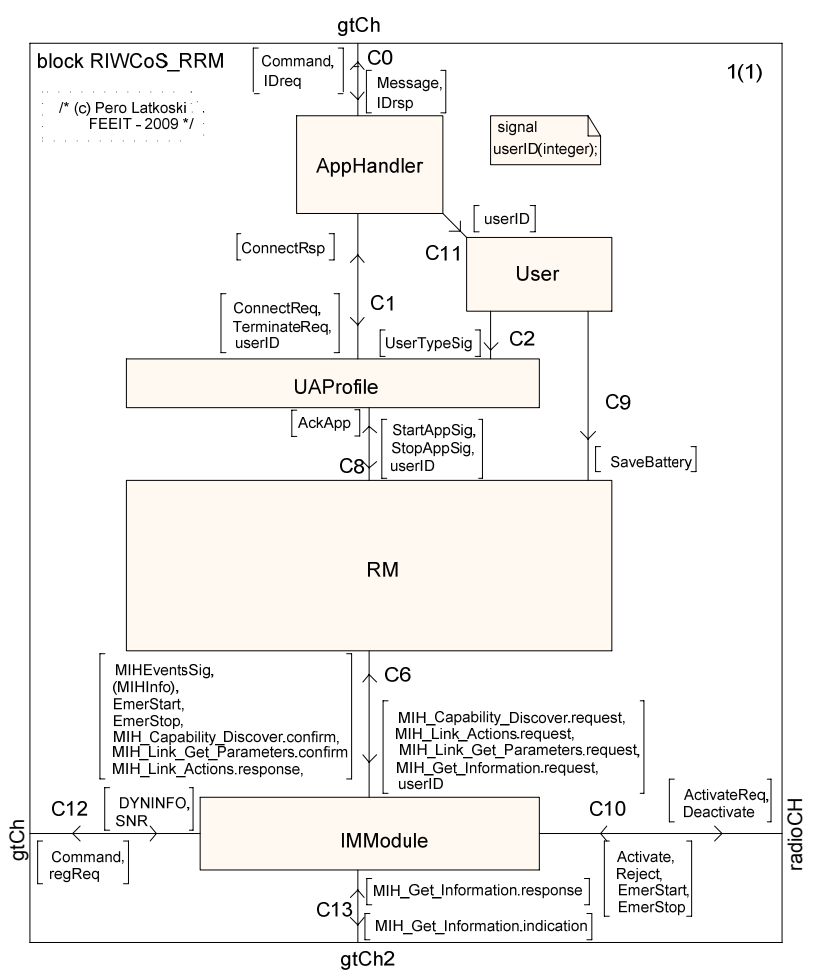

Figure 3. RIWCoS RRM model (block level)

each other. Each instance has a unique identifier (SDL PID). The communication between RIWCoS system and its environment is established through a single SDL channel, Cout. A special block Gateway is used as a router for all incoming and outgoing messages. The Gateway holds information for RRM instances (i.e. PID and instance number), as well as a knowledge of the routes to other SDL model entities.

Inside the RIWCoS RRM block we have build the entire resource manger architecture (Figure 3). The application handler, user details, user\&application profile, resource manager and media independent module are created as separate SDL blocks. Protocol layer entities are modeled as SDL processes, inside the SDL blocks.

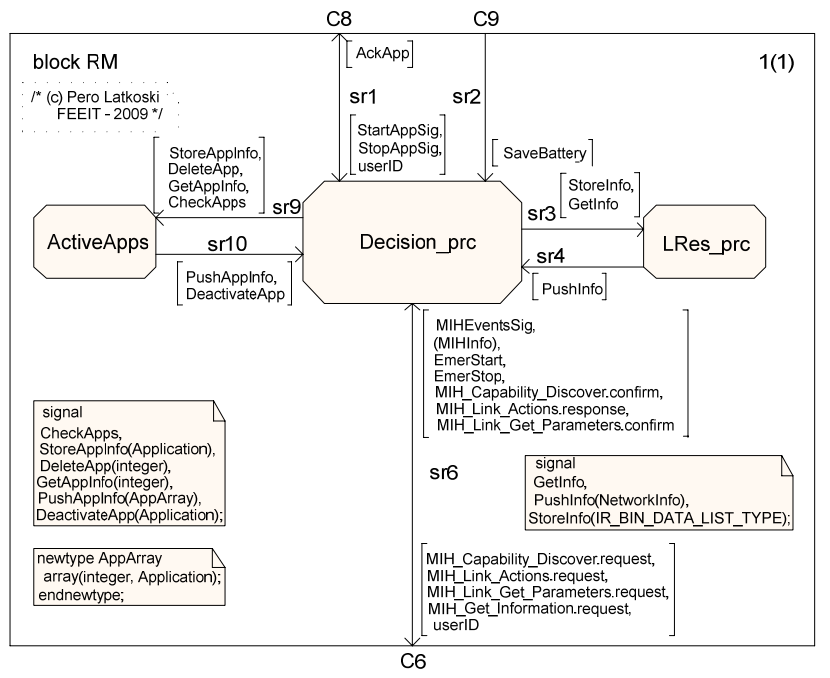

Figure 4. Decision process and its surrounding

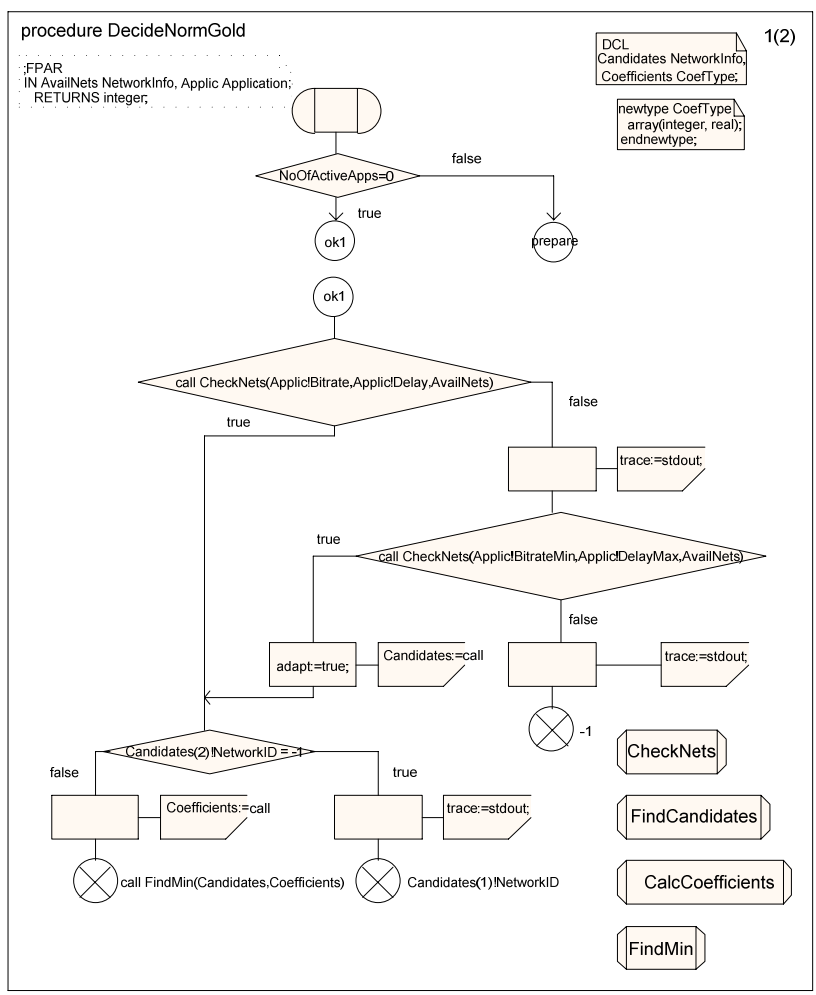

Figure 5. Decision procedure for Normal mode and Gold user

Figure 4 depicts the organization of the SDL model, inside of the RM block. As it can be seen, the decision process utilizes two local repositories: one for the available network links (LRes) and one for the ongoing applications (ActiveApps). In each process an extended communicating finite state machine (ECFSM) is used to specify the behavior of the protocol entity. Figure 5 presents a part of the decision process, in case of a Gold type of user and Normal network's mode of operation. The SDL part of RIWCoS model was created using the tool provided by [28]. This simulation and modeling tool provides extensive automated debugging and testing methods for verification of the 


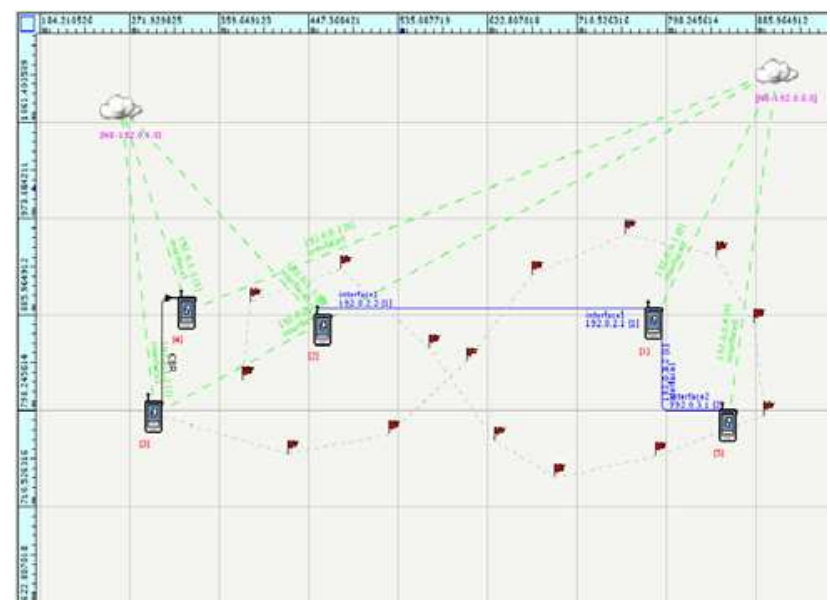

Figure 6. QualNet scenario example

protocol model correctness. Then the SDL sequences, which are initially created by using the graphical representation of SDL (SDL-GR), are converted into SDL phrasal representation (SDL-PR).

The tool translates the SDL-PR code into a $\mathrm{C}++$ code and the compiler builds an executable file. The result is a standalone application which communicates with the QualNet simulator through an interface, as explained in details in the Section 3.3.

\subsection{QualNet Simulator}

The simulation platforms are often considered as a powerful tool in the area of telecommunications' research. A variety of simulators can be found, focusing on different aspects such as wireless propagation, RF planning, network behavior, etc. OPNET [29], NS-2 [30], OMNeT++[31] are network simulation tools which are widely used by the academic community in the area of telecommunications. In this paper the focus is put on the QualNet network simulator. QualNet is an event driven simulation tool that is able to predict the performance of wired, wireless and hybrid network platforms with a high level of precision. It has been developed from its predecessor, GloMoSim (Global Mobile Information System Simulator) network simulator [32]. QualNet is based on a layered model very similar to the OSI layered model.

The main advantages of QualNet are: Good and up-to-date wireless (Physical and Medium Access Control layers of different technologies, channel propagation models, etc.) models; A modular, layered protocol stack that eases the implementations of new protocols; Comparative performance and evaluation of given protocols on different layers, due to the built-in measurements on each layer; GUI (Graphical User Interface) that enables rapid and easy system and network modeling; High level of scalability. A wide range of network architectures can be simulated, including variable number of network nodes.

QualNet's main task in the RIWCoS model is to simulate its platform, instead of a real testbed implementation. The general idea is to use QualNet as a wireless heterogeneous network environment, utilizing variety of technologies such as IEEE $802.11 \mathrm{a} / \mathrm{b}$, IEEE $802.16 \mathrm{~d} / \mathrm{e}$, UMTS, etc. An example of QualNet defined WHN model is depicted in Figure 6.

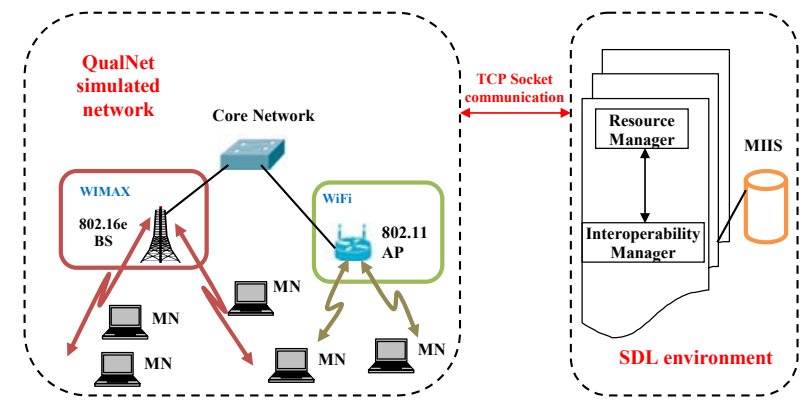

Figure 7. Simulation setup

\subsection{SDL2QualNet Interface}

The communication between QualNet and SDL is done via sockets. The usage of socket communication enables QualNet and SDL to be implemented on different physical machines (Figure 7). This is a benefit in terms of system performance i.e. processing performance and regarding the availability of operating systems (OSs). This means that the external application (SDL) and QualNet can be installed on different platforms and still work properly. Additionally, not always the same OS is possible to be used for the external application and the simulator. A common client - server communication is used between QualNet and SDL. In this case QualNet is the server, while SDL application acts as the client. Because the nature of the communication is based on client-server paradigm, QualNet must be initiated first. It waits for the SDL connection request on a predefined port.

After the SDL sends the connection request they exchange information about the sockets that will be used and the communication starts. The connection stays open throughout the entire simulation. TCP is used as a transport protocol because of its reliable nature. Loosing information can have huge impact on the simulation fidelity, and must be taken into consideration.

The communication between the pure SDL system and its OS physical environment is not direct, but through a C++ Environment specially created for this purpose. The environment function provides translation of the signals received from QualNet server into SDL recognizable signals. When a message is found in the incoming buffer, the environment function identifies the message type and proceeds with the parsing process of the message's parameters. The format of each message type is predefined, i.e. the function and format of all parameters is known. Consistently, every parameter value is cast into an appropriate value type (integer, real, etc.). The received and converted message along with its parameters is then flushed into the SDL system. The protocol which is previously specified in SDL takes over the control of signal and parameters processing. The result of the processing is returned as a SDL signal to the $\mathrm{C}++$ environment. The SDL message and its parameters are translated of into an appropriate message suitable for QualNet, and sent to the QualNet side by using the predefined socket.

The previously presented simulator performed the testing scenario in real time and with a high fidelity. There was no lost of packets, nor buffer congestion. We were able to produce a reach trace file of events, both from SDL and QualNet part. 


\section{WHN IMPLEMENTATION ISSUES}

In order to create a high performance simulation platform the following implementation issues were taken into consideration: dynamic allocation of memory, commanding QualNet nodes from SDL, collecting dynamic network information updates, providing link quality information updates, buffer management and flow control.

Dynamic allocation of memory: The dynamic allocation of memory is one of the most important aspects when modifying QualNet. Due to the structure of the simulator, every node in the simulation has to activate its one class/structure of parameters to store the needed information about its behavior. This means that pointers must be used when accessing the node's parameters for efficient work of the simulator.

Commanding QualNet nodes from SDL: Another crucial issue is the enablement of the handover decision messages that are sent from RRM (SDL) and arrive in QualNet through the external interface. Due to the fact that any node can have multiple applications that can be served through different access networks, in the parameters class of the given node a set of variables should be defined for proper management of the applications on each interface. "Forcing" the mobile node to initiate a handover to a given Point of Attachment is also an important task. To initiate the handover the mobile node has to reconfigure its protocol stack. This means that a set of parameters from all protocol layers should be preset in terms of proper initiation of the handover. One part of the parameters is locally updated, while the other part is sent from the RRM (SDL) in the handover decision message.

The dynamic information update: The dynamic information update is vital for the handover decision. These updates depict the momentary situation of the PoAs in terms of free network capacity, end-to-end delay, and jitter. Due to their dynamical nature, the updates are sent from QualNet to SDL on a periodic basis. Choosing which updates to be sent and when, can be a complex task. If updates are sent too often then problems with buffer management and processing overload of the RRM application occur. If the updates are scarce then the information sent to the RRM is outdated and can result in wrong handover decision. In the simulation scenarios of our interest it has been proven that if the updates are sent in the interval between $0.3 \mathrm{~s}$ and $0.7 \mathrm{~s}$ an optimal solution is achieved, in terms of frequency of transmitted updates and the relevance of the PoAs dynamic change. Different approaches and methods are used for estimating the available network capacity in WiFi and WiMAX PoAs. In WiFi the available capacity is estimated by calculating the ratio between the time that the AP spends in idle (idle or sensing) mode and the total time spent in active (transmitting and receiving) and idle mode. In terms of WiMAX the calculation is based on the Physical Slots (PS). The physical slots are the basic time unit in the IEEE 802.16 standard, and are correlated with the system symbol rate and can also be used for calculating the free network capacity. Mapping the number of PS in OFDM symbols gives the free capacity of the network in OFDM symbols per frame.

Link quality information updates: Updates are also provided for QualNet's mobile nodes. These updates carry out the information about the SNR on every interface. This information is vital for the IEEE 802.21 events triggering and for calculating the available bitrate that can be provided from a given PoA to the mobile user. The free network capacity only gives information about the PoA's capability to serve new users. Proper mapping should be done from the free network capacity to available user bitrate by taking the channel conditions in consideration. Given the received SNR of the WiMAX BS in the mobile node, the RRM can map the number of bits per OFDM symbol. In this way the RRM estimates the available bitrate for the given node. The estimation of the available bitrate in WiFi is similar to the WiMAX estimation: the RRM maps the available user bitrate from the received SNR and the available capacity by using a predefined LOOKUP table in its memory. The values in the table are dynamically updated during the simulation, in which way we increase its precision.

To provide a best $Q o S$ for the mobile users, the RRM receives updates from every application, regarding the bitrate that has been provided and the delay experienced in the given PoA. If the provided bitrate and delay are below the expected values, the RRM can initiate a handover to another PoA. In QualNet the statistics can be only calculated in the sink node. This means that the sink node sends updates about the application performance to the RRM. This kind of updates are sent only once per PoA. It means that after the mobile node has connected to a given PoA, its sink node start to collect application information and after a given time interval it sends the information to the RRM. Because the sink node is not aware of his source when it changes the PoA, the sink must be triggered to start with the acquisition of the given application statistics. The trigger is produced by the RRM.

Buffer management and flow control: The buffer management is crucial in the communication between QualNet and SDL. A single SDL application is being used for all nodes in the simulation scenario. Because of the centralized approach, a huge number of messages are sent from QualNet to SDL in very short time intervals (in terms of micro seconds). These messages are often seen on the receiver side as one large message. This can easily cause an overflow of the SDL buffer. To avoid this problem, the length of the buffer is set to a fixed value of bytes. The same amount of bytes is sent from QualNet each time a message is sent. Regarding the original message length, appropriate amount of padding bytes are added to the message, in order to achieve the predefined message length. In this manner only one message at a time can be read from the SDL buffer. Another problem in terms of buffers in socket programming is the out of sequence reception of fragments when using TCP. When sending larger packets, fragments may arrive out of sequence due to the congestion or TCP retransmission. This can evoke misinterpretation of the received messages at the SDL side. To mitigate the effects of out of sequence reception, on QualNet side a filter must be implemented. The goal of the filter is to allow the transmission of only the messages that are crucial for proper working of the QualNet - SDL simulation. For example, take in consideration the SNR update messages. Only the messages from the mobile nodes need to be sent. The SNR updates taken at the base stations side are not sent. Also, for a given node only the SNR updates that have significant differences in their value in comparison to the previous values are sent. This means that if the SNR update has a value of the SNR that is almost the same 
as the previously sent value, the message would not be sent. By implementing these solutions for buffer management, the communication between QualNet and SDL is kept stable and reliable even for scenarios with extremely large number of nodes with a significant amount of useful and redundant information.

\section{MODEL EVALUATION}

The evaluation of the simulation platform and model is performed with the following scenario setup: the network consists of one WiMAX BS, one WiFi AP and a multi-interface mobile node. The node movement is based on a user predefined mobility model as given in Figure 6 with constant velocity of $10 \mathrm{~m} / \mathrm{s}$. When the simulation is started the mobile node is located in the WiMAX cell. It starts to move and it enters the WiFi zone leaving the WiMAX coverage. In such a way we enforce a VHO (form WiMAX to WiFi) which is need to be initiated for the mobile user to stay active and attached to network. The simulation duration is $100 \mathrm{~s}$. CBR is used as the user application, emulating a real time application (voice and video application). The voice application is a $64 \mathrm{kbps}$ application with frame size of $160 \mathrm{~B}$ and frame duration of $20 \mathrm{~ms}$ [33]. The video is defined as a $640 \mathrm{kbps}$ application with a frame size of $1600 \mathrm{~B}$ and frame duration of $20 \mathrm{~ms}$. In the following we present the model evaluation results obtained from the trace files for the RM performance and the network conditions acquisition.

\subsection{Handover performance analysis}

The RRM model in the simulation triggers the handover providing a decision based on the underlying radio and the application requirements on the terminal. When defining the threshold that will initiate a vertical handover (VHO) many parameters should be taken in consideration. One of the most important is the quality of the radio link. Lack of radio quality can result in degradation of the application performances. On the other hand, the threshold should be set with consideration for the application parameters: average end-to-end delay of the session, average jitter of the session, throughput of the session.

In our scenario, the handover trigger generation is based on the signal quality of all available PoAs. The handover is to be initiated whenever the SNR crosses a predefined threshold. Fig. 8 depicts the monitored value of the SNR at the mobile node, for both PoAs. A trigger Link Going Down is generated when the WiMAX SNR value crosses the threshold of $12 \mathrm{~dB}$. The future work here will introduce an intelligent prediction of the SNR values, in order to prepare the handover decisions on time.

Figure 9 depicts the average end-to-end packet delay for the case when the RRM is used and vertical handover is performed, and the case when there is no handover hence the session continues on the same access network (WiMAX). The average end-to-end delay is measured periodically in time intervals of 1 second during the whole simulation time. As the mobile user moves away from the WiMAX BS the delay increases and shows notable variations. This causes performance degradation for the application. When the RRM initiates a handover decision for the mobile node to connect to the WiFi AP the delay increases. However, when the mobile node stays connected to WiMAX (no handover is initiated) the delay has larger variations with smaller magnitude compared to the delay

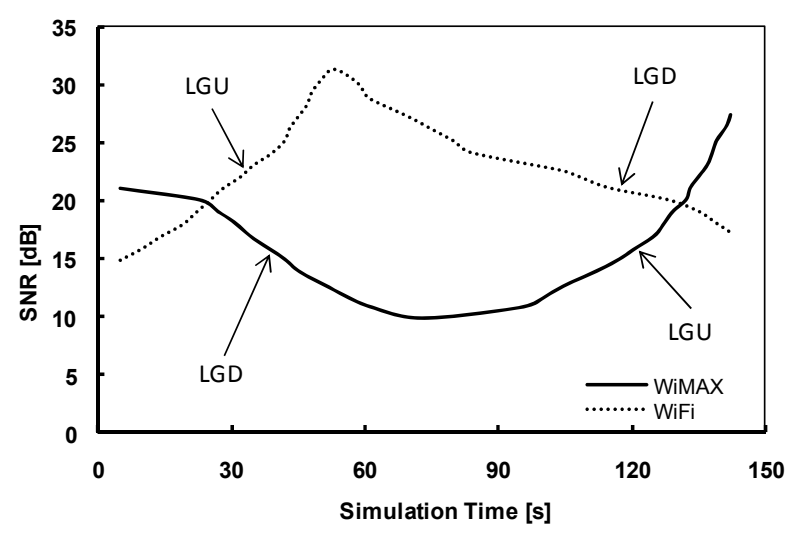

Figure 8. Monitoring of SNR

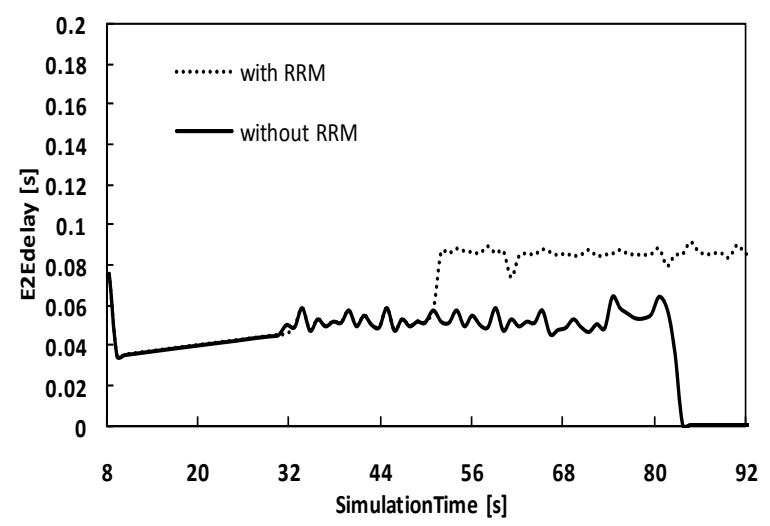

Figure 9. Average end-to-end delay, with and without a HO

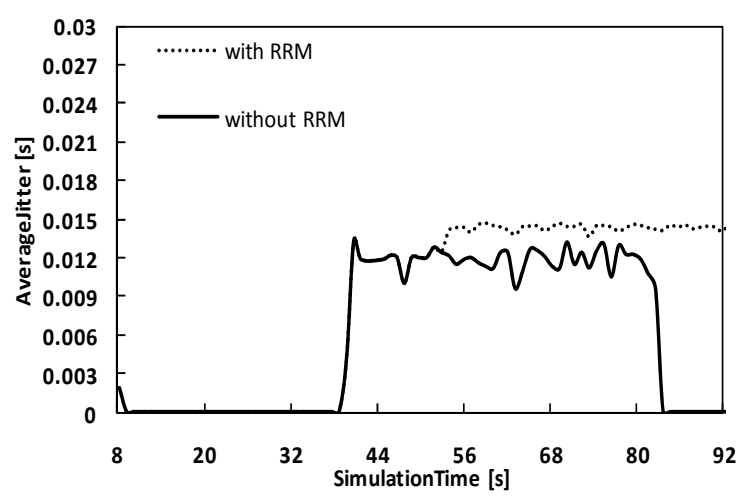

Figure 10. Average Jitter, with and without a HO decision

experienced on WiFi network. The difference in the values of the delay is due to the different MAC layers of the both technologies. In WiMAX there is a more efficient MAC layer compared to WiFi MAC layer. If the RRM handover decision is not used, the user will lose its' connection eventually as it will move outside the WiMAX cell. The RRM should take in consideration that WiMAX has better performance in comparison to $\mathrm{WiFi}$ in order to trigger the handover just before the WiMAX connection is lost.

Similar conclusions can be made if the average jitter is investigated. The results are depicted in Fig. 10. The average 


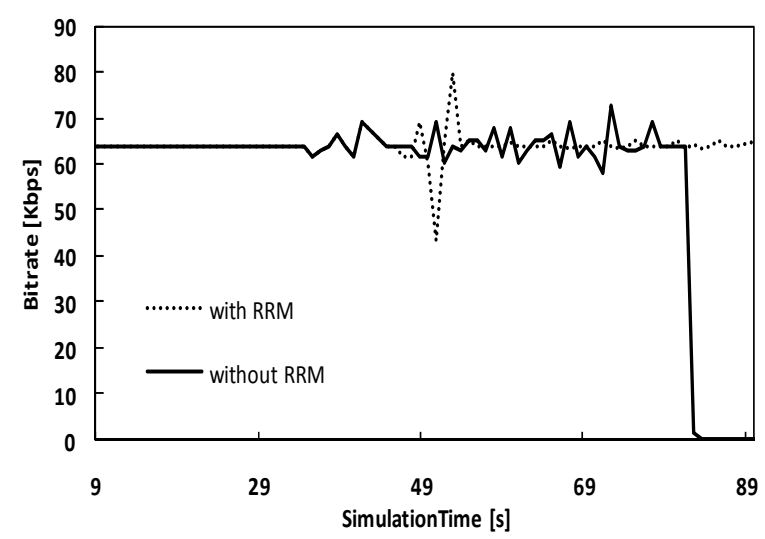

Figure 11. Bitrate behavior, with and without a HO decision

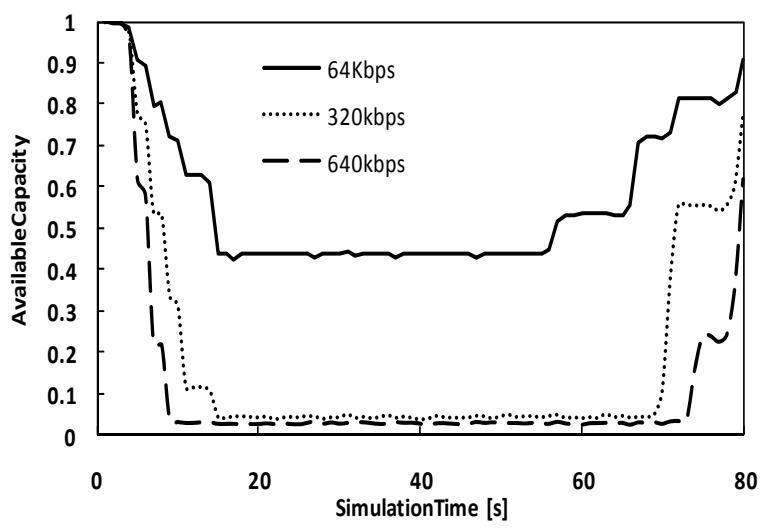

Figure 12. Available Capacity of an IEEE 802.11 AP

jitter is calculated for packets that are received in a time window of 1 second. The values obtained for the jitter between the two approaches do not differ significantly as in the case when the average delay was investigated. In terms of the jitter the radio access technology selection has smaller influence compared to the delay which is significantly influenced by the radio.

Figure 11 depicts the bitrate behavior for the same simulation scenario. Better performance is obtained when the handover is initiated. If the mobile node stays connected to the WiMAX Base Station the bitrate starts to oscillate as the mobile node moves away from the BS. This will result in decrease of the application quality. This fact contrasts the previous case when the delay and the jitter yielded better performance when no handover decision was issued. This means that the SNR threshold set by the RM for triggering the handover should be set to higher values in order for the terminal to connect sooner to the WiFi. Different application configurations will result in different threshold settings to be used when initiating a VHO. If a real time application is used than the delay and jitter have to be considered as primary input parameters for setting up the threshold for the handover. If it is a non-real time application like FTP, than the bitrate will be considered as the primary input parameter for the handover threshold.

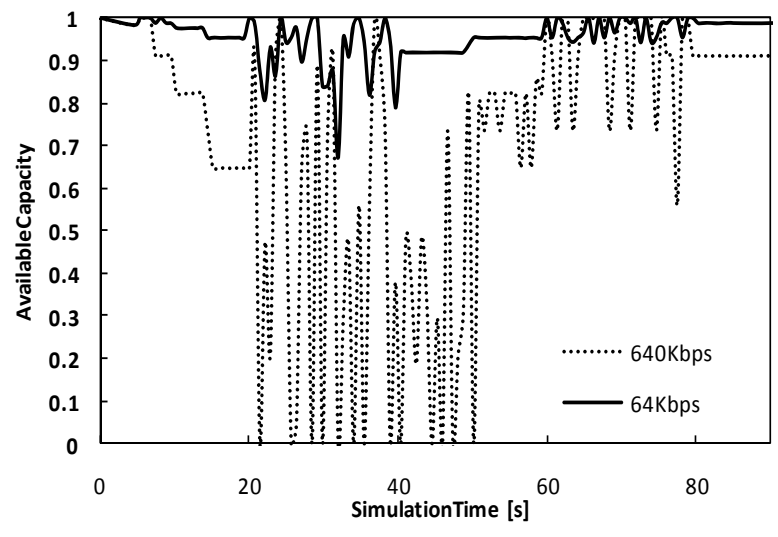

Figure 13. Available Capacity of an IEEE 802.16 BS

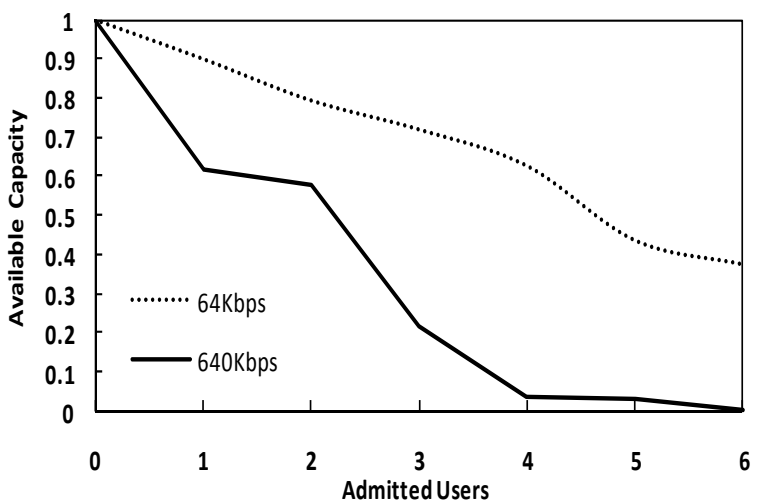

Figure 14. Available Capacity of an IEEE 802.11 AP for different number of users

\subsection{Dynamic updates}

This subsection shows the SDL2QualNet communication in terms of MIH Commands utilization for obtaining information from the network that change dynamically. The MIH user (in this case the RRM placed in SDL) initiates commands for collecting sets of dynamic parameters (e.g. available capacity, average end-to-end delay, packet loss in the network, jitter, etc.) from a given PoA. Figure 12 depicts the available capacity of an IEEE 802.11b Access Point during the simulation time. The available capacity of the given AP is used for determining the bitrate that the networks can offer to any application. The available capacity is normalized in such manner that value 1 corresponds to an AP that does not serve any users at the time and has all it's capacity available.

In this scenario, six mobile terminals enter and exit the coverage of the AP. Each mobile terminal has the same active application. In the scenario, each mobile node enters and exits the AP coverage in different time so the change of the available capacity are easily noticed and better visualized. The available capacity is depicted for three different applications, 64kbps, $320 \mathrm{kbps}$ and $640 \mathrm{kbps}$. When using the $64 \mathrm{kbps}$ application the available capacity of the AP is largest. On the other hand, when the terminals are configured with the $320 \mathrm{kbps}$ and the $640 \mathrm{kbps}$ applications, large portion of the available capacity is consumed and the AP is easily overloaded. It is interesting to note, that 


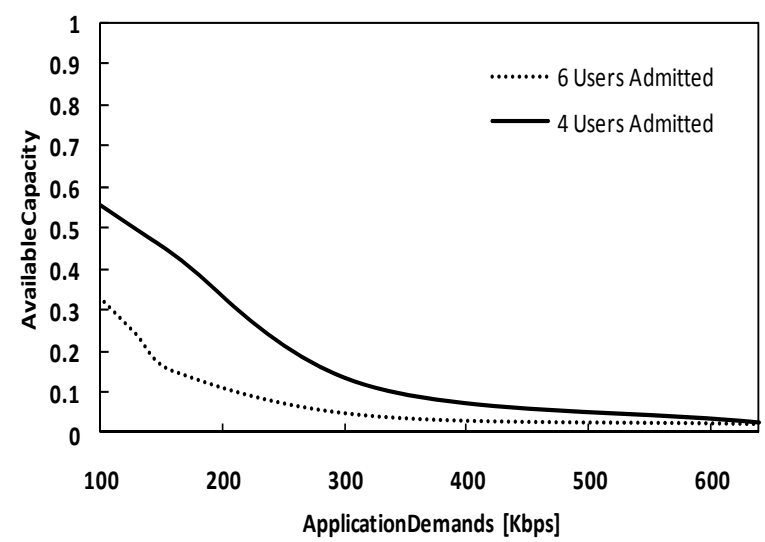

Figure 15. Available Capacity of an IEEE 802.11 AP for different application demands

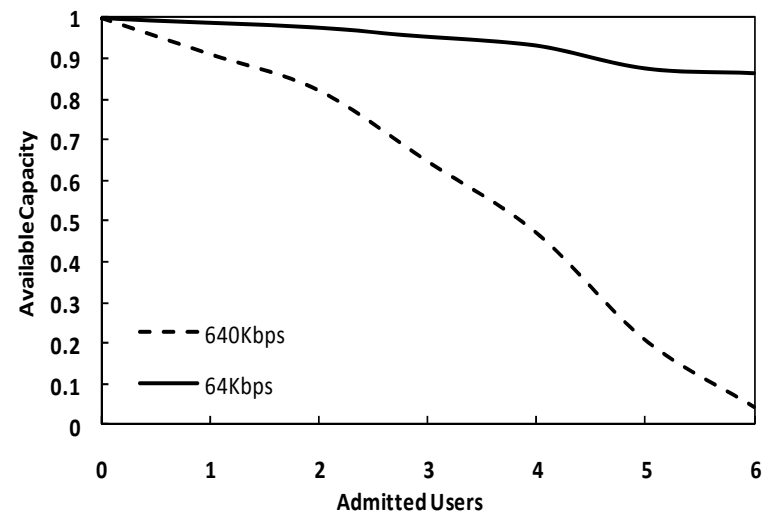

Figure 16. Available Capacity of a WiMAX BS for different number of users

these two types of application, although differ by factor 2, they consume almost the same amount of the capacity.

The same scenario setup was used for evaluating the available capacity in an IEEE 802.16 Base Station (BS). The available capacity is defined through the number free Physical Slots (PS). The PS is a basic time unit in the IEEE 802.16 standard. The PS duration is related to the system symbol rate and can also be used for calculating the free network capacity. The available capacity is normalized in the same manner as the WiFi case.

The available capacity is defined as the ratio between the number of free PSs and the total number of PSs. From Figure 13, it can be noted that when new users connect to the BS the available capacity decreases. For certain dynamic situations (e.g. when admitting new users) the behavior of the available capacity at the BS has an irregular pattern, because it serves the mobile users in bursts. The peak values of the variations are larger when the BS serves users with larger application demands.

As the number of users and the application demands have nonlinear influence over the PoA's performance, hereafter we investigate this influence. Fig. 14 shows the available capacity of the WiFi as a function of the admitted users in the hotspot.

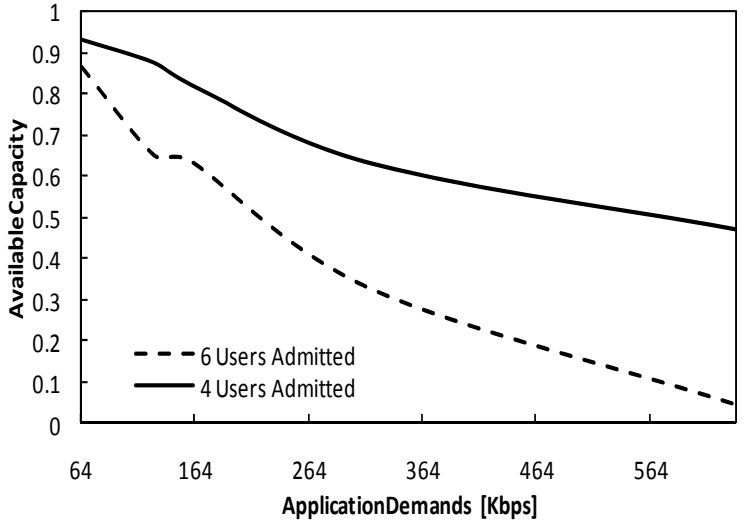

Figure 17. Available Capacity of a WiMAX BS for different applications

When the applications of the admitted users in the terminal are less bandwidth demanding (e.g. 64kbps), then the slope slowly drops as the number of admitted users increases. In contrast to this case, when the applications of the admitted users are with high demands (e.g. 640Kbps) then the curve rapidly drops for smaller number of users.

Fig. 15 shows the available capacity of the WiFi as a function of the application configuration for 4 and 6 admitted users. For larger number of users the drop is more significant even for application that are not resource demanding (e.g. in range of $64 \mathrm{kbps}$ to $300 \mathrm{kbps}$ ). This means that the AP suitability should be estimated depending on the started applications on the terminal which AP already serves.

Fig. 16 shows the available capacity of the WiMAX base station as a function of the number of users that it already servers. Depending on the application demands of the users, the available capacity differs. As the number of users increases, the capacity decreases. This decrease is more notable for application with higher bandwidth demands in contrast to application with lower demands, in which case the drop of the available capacity is slow and gradual.

Fig. 17 depicts the available capacity of the WiMAX station as a function of the application configuration for two cases. In the first case there are 4 users admitted in the network and in the second there are 6 users. For both cases, the higher application configuration causes lower available capacity for the potential users. This drop in capacity is more significant for larger number of users where the available capacity curve drops significantly.

The previously presented results prove that the proposed simulation platform is an efficient tool for modeling and evaluation of wireless heterogeneous networks. All necessary data for proper operation of the radio resource manager were successfully provided from QualNet environment. We have also proved that the network performance can be successfully estimated, by evaluating the effects of an imminent vertical handover simulated in the scenario. All collected performance results are valuable for appropriate configuration of the RRM parameters in more complex and realistic simulation scenarios. 


\section{CONCLUSION}

This paper presented a state-of-the-art simulation platform as a unique combination of QualNet simulation scenario builder and Specification and Description Language protocol prototyping tool. Each part of the proposed combined methodology is specialized in fulfilling a particular simulation and modeling task. The utilized approaches are complementary and the result of the newly proposed bonding is a very powerful platform, specialized for large scale network modeling.

By employing this simulation platform, the concept of comprehensive heterogeneous wireless network model based on the RIWCoS architecture was built and analyzed. Implementation solutions for IEEE 802.21 were presented and additionally evaluated. The paper proposes methods for link quality monitoring, MIH events triggering, and calculation of dynamic link parameters for $\mathrm{WiFi}$ and WiMAX networks. Additional valuable information is provided regarding the QualNet and SDL model implementation and simulation optimization.

The future work will include a complete implementation of the RIWCoS proposed paradigm in emergency conditions, along with an intelligent prediction of link events, such as Link Going Down, and the concerns of terminal's battery consumption towards the green radio paradigm.

\section{ACKNOWLEDGMENTS}

This research is sponsored by NATO's Public Diplomacy Division in the framework of "Science for Peace" through the SfP-982469 "Reconfigurable Interoperability of Wireless Communications Systems (RIWCoS)" project.

\section{REFERENCES}

[1] V. Atanasovski and L. Gavrilovska, "Managing Emergency Situations in IEEE 802.21 Enabled Networking Environments," International IEEE Conference EUROCON 2009, Saint-Petersburg, Russia, May 2009.

[2] A. Hall, "Seven Myths of Formal Methods," IEEE Software, vol. 7, no. 5, Sept. 1990, pp. 11-9.

[3] Thomas Kuhn, Alexander Geraldy, Reinhard Gotzhein and Florian Rothländer "ns + SDL - The Network Simulator for SDL Systems", LNSC Volume 3530/2005.

[4] K. G. Larsen, P. Pettersson, and W. Li, "UPPAAL in a Nutshell," Springer Int'l. J. Software Tools for Technology Transfer, vol. 1+2, 1997.

[5] P. Latkoski, L. Gavrilovska, "Analysis of Bluetooth Protocol in Presence of Bursty Traffic", Journal of Communications - JCM, Academy Publisher, issue 6, December 2007.

[6] Glenford Mapp, at all, "An Architectural Framework for Heterogeneous Networking", International Conference on Wireless Information Networks and Systems (WINSYS), August 2006.

[7] Christian M. Mueller, Harald Eckhardt, Rolf Sigle, "Realization Aspects of Multi-Radio Management Based on IEEE 802.21”, LNCS, Springer, May, 2009.
[8] O. Ognenoski, V. Atanasovski, P. Latkoski, L. Gavrilovska, "Resource Management Aspects in Future Wireless Systems: RIWCoS Approach", 2nd International Symposium on Applied Sciences in Biomedical and Communication Technologies - ISABEL 2009, Slovakia.

[9] C.A.Petri, "Nets, Time and Space", Theoretical Computer Science, 1996, No. 1-2, Vol. 153, Pages: 3-48.

[10] Joachim Sachs, Mikael Prytz, Jens Gebert, "Multi-access Management in Heterogeneous Networks", Journal of Wireless Personal Communications, Springer, 2007.

[11] Pengbo Si, Hong Ji, F. Richard Yu, "Optimal network selection in heterogeneous wireless multimedia networks", Journal of Wireless Networks, Springer, August 2009.

[12] Yi Sun, "Automatic Flow Distribution and Management in Heterogeneous Networks", GLOBECOM 09, USA, 2009.

[13] D. Tsamis, at all, "Dynamic resource modeling for heterogeneous wireless networks", IEEE International Conference on Communications (ICC), Germany, 2009.

[14] J. M. Wing, “A Specifier's Introduction to Formal Methods," IEEE Computer, vol. 23, no. 9, Sept. 1990.

[15] Project RIWCoS - Reconfigurable Interoperability of Wireless Communications Systems (NATO funding under SfP-982469). http://riwcos.comm.pub.ro

[16] IEEE 802.21: Media Independent Handover, http://www.ieee802.org/21.

[17] ITU-T, Recommendation Z.100 (11/99) Specification and Description Language (SDL).

[18] Information Processing Systems - OSI: Estelle, A Formal Description Technique Based on an Extended State Transition Model, International Standard 9074, June 1989.

[19] ISO/IEC JTC1/SC7, International Standard 15437: Information technology Enhancements to LOTOS (ELOTOS), July 2000.

[20] ITU-T, Recommendation Z.120 (MSC), Nov. 1999.

[21] OMG Unified Modeling Language Specification, Version 1.3, March 2000.

[22] ITU-T, "Specification of abstract syntax notation one (ASN.1)," ITU- T Reconunendation X.208, Technical Report, Telecommun. Standardization Sector of ITU, 1993.

[23] ITU-T, Recommendation Z.140 Tree and Tabular Combined Notation (TTCN), March 2006

[24] http://netlib.sandia.gov/spin/index.html

[25] http://www.etsi.org

[26] http://www.3gpp.org

[27] http://www.ieee.org

[28] http://www.telelogic.com

[29] http://www.opnet.com

[30] http://www.isi.edu/nsnam/ns

[31] http://www.omnetpp.org

[32] http://pcl.cs.ucla.edu/projects/glomosim

[33] http://www.itu.int/rec/T-REC-G.7 